\title{
Dietary methods and biomarkers of omega 3 fatty acids: a systematic review
}

\author{
Lluis Serra-Majem ${ }^{1,2 *}$, Mariela Nissensohn ${ }^{1}$, Nina C. Øverby ${ }^{3}$ and Katalin Fekete ${ }^{4}$ \\ ${ }^{1}$ Department of Clinical Sciences, University of Las Palmas de Gran Canaria, PO Box 550 35080, Las Palmas de Gran \\ Canaria, Spain \\ ${ }^{2}$ Community Nutrition Research Centre of the Nutrition Research Foundation, University of Barcelona Science Park, Baldiri \\ Reixac 4, 08028 Barcelona, Spain \\ ${ }^{3}$ Faculty of Health and Sport, University of Agder, Service Box 422, 4604 Kristiansand, Norway \\ ${ }^{4}$ Department of Biochemistry and Medical Chemistry, University of Pécs Szigeti út 12., H-7624 Pécs, Hungary
}

\begin{abstract}
The aims of the present study were to review the validity of dietary methods used to measure the usual long chain (LC) omega- 3 polyunsaturated fatty acid ( $n-3$ PUFA) intake of a population and to assess the usefulness of different biomarkers of $n$-3 PUFA in healthy humans. Two systematic literature searches were conducted until May 2011 to update previous systematic reviews. The first literature search aimed to find studies validating the methodology used for measuring the dietary intake of $n$ - 3 PUFA. The second search aimed to find human intervention studies in which $n-3$ PUFA status changed after 2 weeks of $n-3$ PUFA supplementation. Sixteen studies were identified for inclusion in the first review. Correlation coefficients between fatty acids in subcutaneous fat or blood lipids and dietary intake of $n$-3 PUFA from different questionnaires were similar. Subcutaneous fat has been reported as the best reference method for some authors, and these studies showed moderate correlation coefficients with no dietary intake method being superior to any other. As for the evaluation of biomarkers of docosahexaenoic acid (DHA, 22:6n-3) and eicosapentaenoic acid (EPA, 20:5n-3) status in response to supplementation, the new search reaffirmed and reinforced the evidence supporting that plasma phospholipid DHA, erythrocyte DHA, and platelet DHA were all effective and robust biomarkers of DHA status. Our findings only confirmed earlier studies and did not provide evidence for reaching new conclusions.
\end{abstract}

Key words: Polyunsaturated fatty acids: Omega-3: Dietary intake: Nutritional status: Dietary methods: Biomarkers

\section{Introduction}

Nutritional epidemiological research requires addressing issues of measurement errors and inter and intra-individual variability, which are specific for each nutrient. Public health decisions must rely on valid and precise estimates of nutrient intake and status. There is a need to reach a consensus about the best available methods for assessing nutrient intake and status at the population level. In this article a literature review of dietary methods used to assess intake of $n-3$ PUFA is presented. Also biomarkers for $n-3$ PUFA status were analysed. The effect of dietary fats on health and disease has been of interest for many decades. The various health benefits of consuming the LC $n$-3 PUFA particularly eicosapentaenoic acid (EPA, 20:5n-3) and docosapenteanoic acid (DHA, $22: 6 n-3)$, have been widely reported ${ }^{(1)}$. The LC $n-3$ PUFA are obtained predominantly from fish, seafood, meat, and eggs ${ }^{(1)}$. However, various dietary supplements containing several hundred milligrams of LC $n-3$ PUFAs per dose are commonly available. Many clinical studies have assessed the effect of LC $n$-3 PUFA supplementation in restoring health and maintaining well-being. The majority of these reviews concluded that, although there was some indication of the beneficial effect of LC $n-3$ PUFA supplementation, further studies were needed to establish efficacy of their use. To date, there is lack of a universally accepted biomarker that reflects increased LC $n$-3 PUFA status in response to increased dietary intake or supplementation. It is even more important in epidemiologic studies assessing health effects of LC $n-3$ PUFA status in populations over a long-term period to understand which biomarkers truly reflect LC $n-3$ PUFA status. To assess the reliability of biomarkers in reflecting LC $n$-3 PUFA intake, it is necessary to review biomarker data from studies reporting a change in LC $n$-3 PUFA status. On the other hand it is also necessary to know the validity and reproducibility of dietary intake estimations of LC $n$-3 PUFA from different questionnaires with regard to the appropiatte biomarkers. Therefore, the aims of this paper were to review the validity of methods used to measure the usual $n$ - 3 PUFA intake of a population and additionally, to assess the usefulness of different biomarkers of LC $n-3$ PUFA status in healthy humans. 


\section{Methods}

This article includes two updated systematic reviews. Both systematic literature searches were performed between March and May 2011. Two previous systematic reviews covering the objectives of both searches were conducted in 2007 and 2009 within the European Network of Excellence EURopean micronutrient RECommendations Aligned (EURRECA) ${ }^{(2)}$.

For the first search updating the validity of methods to assess usual $n$ - 3 PUFA intake, the literature search was conducted in Medline, OvidSP and EMBASE using the following terms: 'omega-3 fatty acid', 'fish oils', 'biomarker', 'nutritional assessment', and 'fat intake' including MESH-terms. In total 286 articles were selected using Medline, 358 were selected from OvidSP and 330 were identified from EMBASE.

To select the articles to be included in the present review the following exclusion criteria were used: (a) studies conducted exclusively in diseased or institutionalised persons, (b) studies relating diseases to food consumption or nutrient intake, (c) intervention studies and other therapeutic studies with nutrients or drugs related to the metabolism of these nutrients, (d) studies in animals, (e) studies written in languages other than English or Spanish, (f) studies using single 24-hour recall or non validated FFQ, (g) studies related to fish consumption, (h) studies in infants and children, and (i) studies using another dietary method as a reference tool.

Nine hundred seventy four titles and abstracts were identified via the electronic search from the three different databases. After excluding duplicate studies, a total of 87 appeared to be potentially relevant, and we attempted to obtain them in fulltext version. The literature lists in the selected papers were checked and consequently 3 more articles were included. From the 90 articles, 8 of them were chosen to update Table 1 elaborated in the original article from $2009^{(3)}$. In total 19 studies were reviewed ( 11 were already in the first review and 8 were consequently added). To assess the quality of the different calibration/validation studies a quality score system was developed $^{(4)}$. This has been described in previous publications by

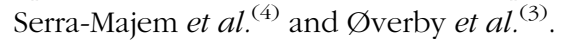

For the second search of the present article aiming to assess the utility of biomarkers for $n-3$ PUFA, another OvidSP and MEDLINE search was developed in order to refresh the search strategy developed by Fekete $e t a l .^{(5)}$ in the systematic review of recovery studies. In this case, the search targeted intervention/recovery studies of $n$-3 PUFA using text terms with appropriate truncation and relevant indexing terms. The following strategy was applied: ( $n$-3 LCPUFA terms) and (intervention study terms) and (human studies) and was limited to the last 4 years. The inclusion criteria was the same as that used by Fekete et al. (2007) $)^{(5)}$. Six hundred and twenty one titles and abstracts were identified via electronic search. From these, only 8 studies were selected to update studies included in the initial study conducted by Fekete $e t a l{ }^{(5)}$

\section{Biomarkers}

Eighteen different biomarkers were used to characterize changes in LC $n$-3 PUFA status. Discussion is only included for those biomarkers used in more than 3 different studies. Data for each study included in the present analysis is described in Table 2. The effects of LC $n$-3 PUFA supplementation on each biomarker are detailed in Table 3 . The main focus is directed towards the effect of DHA supplementation on biomarkers reflecting changes in DHA values. Plasma phospholipid DHA as well as erythrocyte and platelet DHA appear to be reliable and robust biomarkers as shown in Table 3 .

\section{Results}

\section{Dietary method studies}

Details of the 19 papers selected are given in Table 1 . In the 19 articles included in the review, 15 different food frequency questionnaires (FFQ) were validated. All FFQs were designed to capture the usual diet. Some questionnaires specifically asked only about $n$-3-PUFA rich food ${ }^{(1,6)}$, while others covered the whole diet with 66-360 food items included in the questionnaire $^{(7-17)}$. A diet history questionnaire had been validated in one study ${ }^{(18)}$. Weighed records had been validated in 4 studies $^{(12,19,20,21)}$.

In the presented studies the numbers of participants varied from 24 to 4439. The age distribution ranged from 18 to 86 years, with mean ages from 45 to 65 years. In total 15 different FFQs and dietary records or recalls in 5 different settings (varying number of days and season) were validated against subcutaneous fat, serum or plasma fatty acids.

Subcutaneous fat. Adipose tissue fatty acids were determined using chromatography and calculating the area under the curve for each of the fatty acids. All studies using fatty acids in tissue reported the same procedure with only slight modifications ${ }^{(7,8,10,13,14,19)}$.

Five different FFQ were validated against adipose tissue $^{(7,8,10,13,14)}$. All these correlations were significant. Furthermore Marckmann et al. ${ }^{(19)}$ validated weighed records $(3 \times 7 \mathrm{~d})$ against subcutaneous fat. Only DHA crude correlations were significant. Finally Knutsen et $a l .{ }^{(13)}$ validated eight different 24-h recalls of intake of ALA, EPA and DHA against subcutaneous fat. They found high adjusted correlations for ALA, while the correlations for EPA and DHA were lower (Table 1).

Blood component concentrations. After extraction and isolation the serum/plasma phospholipids were quantified by gas liquid chromatography after methylation $^{(6,8,9,11,12,15-18,20-23)}$. Some expressed the serum phospholipids as $\mathrm{mg}$ fatty acid/1 serum ${ }^{(22)}$, while most used percent of total fatty acid methyl esters ${ }^{(8,18,20)}$ or both ${ }^{(11)}$ For detailed descriptions refer to each particular study.

Eleven different FFQ were validated against erythrocytes, plasma or serum ${ }^{(1,6,8,9,11,12,15-17,22,24)}$. Sullivan et al. ${ }^{(1)}$ validated fatty acid estimated from a FFQ against both fatty acid from erythrocytes and from plasma. All the correlations were significant. Andersen et al. ${ }^{(8)}$, Hjartaker et al. ${ }^{(22)}$ and Hodge et $a l^{(11)}$ reported significant correlations of approximately 0.50-0.60 between dietary intake of EPA and DHA estimated from the FFQ and concentrations of EPA and DHA in serum or plasma. Arsenault et al. $^{(9)}$ reported adjusted correlations 


\section{N British Journal of Nutrition}

Table 1. Description of the studies included in this review validating intake of $n-3$ fatty acids (sorted by publication date). Crude and adjusted correlations for dietary methods vs. reference methods in the studies included

\begin{tabular}{|c|c|c|c|c|c|c|c|}
\hline Author & Year pub & Country & Subjects $(n)$ & Age & $\begin{array}{l}\text { Dietary method } \\
\text { which was validated }\end{array}$ & $\begin{array}{l}\text { Reference } \\
\text { method }\end{array}$ & Results \\
\hline \multirow[t]{2}{*}{ Hunter et al. ${ }^{(7)}$} & 1992 & USA & 118 & $45-65$ years & FFQ1 and FFQ2 & $\begin{array}{l}\text { Subcutaneous } \\
\text { fat aspi- } \\
\text { rates from } \\
\text { the lateral } \\
\text { buttock }\end{array}$ & $\begin{array}{l}\text { Crude: FFQ1: EPA: } 0.43 \dagger,{ }^{\star \star \star} \\
\text { FFQ2: EPA: } 0.48 \dagger,{ }^{* \star \star}\end{array}$ \\
\hline & & & & & & & $\begin{array}{l}\text { Energy adjusted: FFQ1: EPA: } 0 \cdot 47^{\star \star \star} \\
\text { FFQ2: EPA: } 0 \cdot 47^{\star \star \star}\end{array}$ \\
\hline Marckmann et al. ${ }^{(19)}$ & 1995 & Denmark & 24 & 20-29 years & $\begin{array}{l}\text { Three } \times 7 d \text { weighed } \\
\text { food records }\end{array}$ & $\begin{array}{l}\text { Fatty acid } \\
\text { composition } \\
\text { of subcu- } \\
\text { taneous fat }\end{array}$ & $\begin{array}{l}\text { Crude: EPA } 0.40 \S \\
\text { DHA } 0.66 \S,{ }^{\star \star \star}\end{array}$ \\
\hline Ma et al. ${ }^{(17)}$ & 1995 & USA & 3570 & $45-64$ years & $\mathrm{FFQ}$ & $\begin{array}{l}\text { Plasma } \\
\text { cholesterol } \\
\text { ester }\end{array}$ & $\begin{array}{l}\text { Crude: ALA: } 0.21 \S \\
\text { EPA: } 0.23 \S \\
\text { DHA: } 0.42 \S\end{array}$ \\
\hline \multirow[t]{2}{*}{ Godley et al. ${ }^{(10)}$} & 1996 & USA & $\begin{array}{l}36 \text { erythrocyte controls, } \\
33 \text { adipose tissue } \\
\text { controls }\end{array}$ & $46-86$ years & $\mathrm{FFQ}$ & $\begin{array}{l}\text { Erythrocyte } \\
\text { membrane }\end{array}$ & $\begin{array}{l}\text { Crude: EPA: } 0.36 \dagger \\
\text { DHA: } 0.19 \dagger\end{array}$ \\
\hline & & & & & & $\begin{array}{l}\text { Adipose tis- } \\
\text { sue }\end{array}$ & $\begin{array}{l}\text { Crude: EPA: } 0.33 \dagger \\
\text { DHA: } 0.42 \dagger\end{array}$ \\
\hline Hjartaker et al. ${ }^{(22)}$ & 1997 & Norway & 234 & $40-42$ years & $\mathrm{FFQ}$ & $\begin{array}{l}\text { Serum phos- } \\
\text { pholipids } \\
\text { fatty acid } \\
\text { composition }\end{array}$ & $\begin{array}{l}\text { Crude: EPA: } 0.58 \dagger, \ddagger,{ }^{* \star \star} \\
\text { DHA: } 0.53 \dagger, \ddagger, * \star \star\end{array}$ \\
\hline Andersen et al. ${ }^{(8)}$ & 1999 & Norway & $\begin{array}{l}119 \text { adipose tissue } \\
\text { samples, } 135 \text { blood } \\
\text { samples }\end{array}$ & $20-55$ years & $\mathrm{FFQ}$ & $\begin{array}{l}\text { Subcutaneous } \\
\text { adipose tis- } \\
\text { sue of the } \\
\text { buttock } \\
\text { Serum fatty } \\
\text { acid }\end{array}$ & 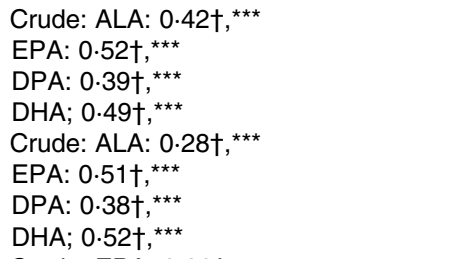 \\
\hline Norrish et al. ${ }^{(16)}$ & 1999 & New Zealand & $\begin{array}{l}480 \text { age-matched } \\
\text { community controls }\end{array}$ & $69.1(\mathrm{SD} 0.3)$ years & $\mathrm{FFQ}$ & $\begin{array}{l}\text { Erythrocyte } \\
\text { phospha- } \\
\text { tidylcholine }\end{array}$ & $\begin{array}{l}\text { Crude: EPA: } 0.26 \dagger \\
\text { DHA: } 0.32 \dagger\end{array}$ \\
\hline Sasaki et al. ${ }^{(18)}$ & 2000 & Japan & 42 men, 44 women & $19-58$ years & $\mathrm{DHQ}$ & $\begin{array}{l}\text { Serum fatty } \\
\text { acids }\end{array}$ & 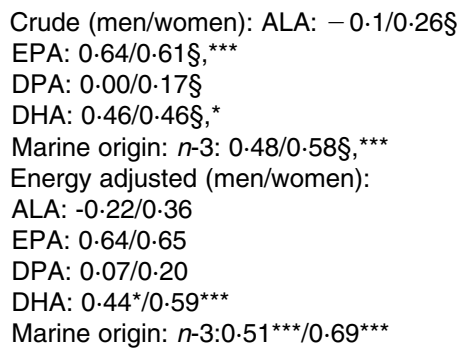 \\
\hline
\end{tabular}


Table 1. Continued

\begin{tabular}{|c|c|c|c|c|c|c|c|}
\hline Author & Year pub & Country & Subjects $(n)$ & Age & $\begin{array}{l}\text { Dietary method } \\
\text { which was validated }\end{array}$ & $\begin{array}{l}\text { Reference } \\
\text { method }\end{array}$ & Results \\
\hline Kobayashi et al. ${ }^{(20)}$ & 2001 & Japan & 87 & $50-59$ years & $\begin{array}{l}7 \mathrm{~d} \text { food weighed } \\
\text { record }\end{array}$ & $\begin{array}{l}\text { Serum phos- } \\
\text { pholipids } \\
\text { fatty acid }\end{array}$ & 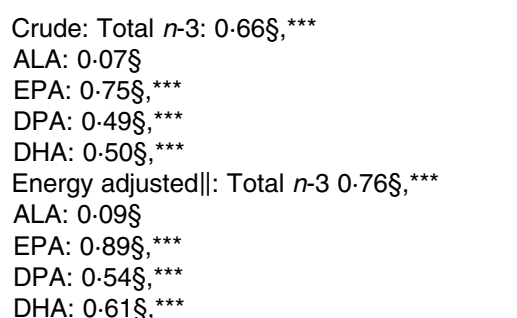 \\
\hline Baylin et al. ${ }^{(14)}$ & 2002 & Costa Rica & 367 men, 136 women & $\begin{array}{l}\text { Men } 56 \text { (SD 11) years, } \\
\text { women } 60 \\
\text { (SD 10) years }\end{array}$ & FFQ & $\begin{array}{l}\text { Adipose } \\
\text { tissue of } \\
\text { the buttock }\end{array}$ & $\begin{array}{l}\text { Crude: ALA: } 0 \cdot 34 \dagger,{ }^{\star \star \star} \\
\text { EPA: } 0 \cdot 15 \dagger,{ }^{\star \star \star} \\
\text { DPA: } 0 \cdot 03 \dagger \\
\text { DHA: } 0 \cdot 18 \dagger,{ }^{\star \star \star}\end{array}$ \\
\hline Kuriki et al. ${ }^{(21)}$ & 2003 & Japan & 15 men, 79 women & $35-55$ years & $7 \mathrm{~d}$ weighed record & $\begin{array}{l}\text { Plasma fatty } \\
\text { acids }\end{array}$ & $\begin{array}{l}\text { Only adjusted presented (men/women): } \\
\text { ALA: } 0 \cdot 35^{\star} / 0 \cdot 19 \\
\text { EPA: } 0 \cdot 57^{\star} / 0 \cdot 60^{\star \star \star} \\
\text { DHA: } 0 \cdot 57^{\star} / 0 \cdot 3^{\star \star *} \text { Adjusted for age and BMI }\end{array}$ \\
\hline \multirow[t]{2}{*}{ Knutsen et al. ${ }^{(13)}$} & 2003 & USA & 49 black and 72 white & $48(S D$ 15.2) years & FFQ & $\begin{array}{l}\text { Adipose } \\
\text { tissue from } \\
\text { the buttock }\end{array}$ & Crude (black/white): ALA: $0.29^{*} / 0.49$ \\
\hline & & & & & $\begin{array}{c}\text { Eight different } \\
24 \mathrm{~h} \text { recalls }\end{array}$ & & $\begin{array}{l}\text { Crude (black/white): ALA: } 0.51 / 0.41 \\
\text { EPA: } 0 \cdot 19 /-0.04 \\
\text { DHA:0.32/0.05 } \\
\text { Energy adjusted (black/white) } \uparrow: \\
\text { ALA: } 0.68^{\star * \star} / 0 \cdot 62^{* \star *} \\
\text { EPA: } 0.23 /-0.05 \\
\text { DHA: } 0.54^{* \star \star} / 0.06\end{array}$ \\
\hline Sullivan et al. ${ }^{(1)}$ & 2006 & Australia & 53 & $19-58$ years & FFQ & $\begin{array}{l}\text { Red blood cell } \\
\text { fatty acids }\end{array}$ & $\begin{array}{l}\text { Crude: Total } n-3 \text { PUFA: } 0.50 \dagger,{ }^{*} \\
\text { EPA: } 0.40 \dagger,{ }^{*} \\
\text { DPA: } 0.05 t \\
\text { DHA: } 0.39 t^{*}\end{array}$ \\
\hline & & & & & & $\begin{array}{l}\text { Plasma fatty } \\
\text { acids }\end{array}$ & $\begin{array}{l}\text { Crude: Total LC } n-3 \text { PUFA: } 0.54 \dagger,{ }^{*} \\
\text { EPA: } 0.54 \dagger,{ }^{*} \\
\text { DPA : } 0.09 \dagger \\
\text { DHA: } 0.48 \dagger,{ }^{*}\end{array}$ \\
\hline Hodge et al. ${ }^{(11)}$ & 2007 & Australia & 4439 & $40-69$ years & FFQ & $\begin{array}{l}\text { Plasma phos- } \\
\text { pholipid } \\
\text { fatty acids }\end{array}$ & $\begin{array}{l}\text { Crude: Total } n-3 \%: 0.31 \dagger \\
\text { ALA: } 0.07 \dagger \\
\text { EPA: } 0.18 \dagger \\
\text { DHA: } 0.4 \dagger \\
\text { Energy adjusted } \dagger \text { : Total } n-3: 0.57 \dagger \\
\text { ALA: } 0.24 \dagger \\
\text { EPA: } 0.40 \dagger \\
\text { DHA: } 0.78 \dagger,\end{array}$ \\
\hline McNaughton et al. ${ }^{(12)}$ & 2007 & Australia & 43 & $28-75$ years & FFQ & $\begin{array}{l}\text { Plasma phos- } \\
\text { pholipid } \\
\text { fatty acids }\end{array}$ & $\begin{array}{l}\text { Crude: Total } n-30.38 \dagger,^{*} \\
\text { ALA: } 0.00 \dagger \\
\text { EPA: } 0.21 \dagger \\
\text { DPA: }-0.05 \dagger \\
\text { DHA: } 0.32 \dagger^{\star}\end{array}$ \\
\hline
\end{tabular}




\section{Nesitish Journal of Nutrition}

Table 1. Continued

\begin{tabular}{|c|c|c|c|c|c|c|c|}
\hline Author & Year pub & Country & Subjects $(n)$ & Age & $\begin{array}{l}\text { Dietary method } \\
\text { which was validated }\end{array}$ & $\begin{array}{l}\text { Reference } \\
\text { method }\end{array}$ & Results \\
\hline & & & & & Weighed record & $\begin{array}{l}\text { Plasma phos- } \\
\text { pholipid } \\
\text { fatty acids }\end{array}$ & $\begin{array}{l}\text { Crude: Total } n-3 \text { PUFA: } 0.33 \dagger,{ }^{*} \\
\text { ALA: } 0.09 \dagger \\
\text { EPA: } 0.22 \dagger \\
\text { DPA: } 0.25 \dagger \\
\text { DHA: } 0.43 t^{*}{ }^{*}\end{array}$ \\
\hline \multirow[t]{8}{*}{ Sun et al..$^{(15)}$} & 2007 & USA & 306 & $43-69$ years & $\mathrm{FFQ}$ & $\begin{array}{l}\text { Plasma fatty } \\
\text { acids }\end{array}$ & 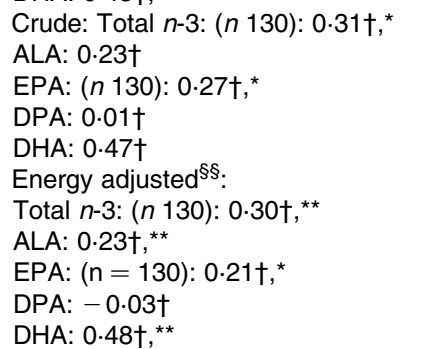 \\
\hline & & & & & & Erythrocyte & $\begin{array}{l}\text { Crude: Total } n-3:\left(n \text { 132): } 0.42 \dagger,{ }^{* *}\right. \\
\text { ALA: } 0.17 \dagger\end{array}$ \\
\hline & & & & & & & EPA: $(n 132): 0.23 t^{* \star}$ \\
\hline & & & & & & & $\begin{array}{l}\text { DPA: } 0.02 \dagger \\
\text { DHA: } 0.54 \dagger\end{array}$ \\
\hline & & & & & & & Energy adjusted ${ }^{\S \S}$ \\
\hline & & & & & & & $\begin{array}{l}\text { Total } n-3:(n 132): 0 \cdot 41 \mathrm{t},{ }^{* *} \\
\text { ALA: } 0 \cdot 18 t^{* *}\end{array}$ \\
\hline & & & & & & & $\begin{array}{l}\text { EPA: }(n 132): 0.38 t^{* \star} \\
\text { DPA: } 0.01 t,{ }^{*}\end{array}$ \\
\hline & & & & & & & DHA: $0.56 \dagger,{ }^{* *}$ \\
\hline Arsenault et al. ${ }^{(9)}$ & 2008 & USA & $\begin{array}{l}129 \text { normal clinical } \\
\text { diagnosis }\end{array}$ & 72.6 (SD 8.0) years & $\mathrm{FFQ}$ & $\begin{array}{l}\text { Plasma phos- } \\
\text { pholipid } \\
\text { fatty acids }\end{array}$ & $\begin{array}{l}\text { Only adjusted presented }{ }^{\prime \prime \prime}: \\
\text { EPA: } 0.38 \S \\
\text { DHA: } 0.49 \S\end{array}$ \\
\hline Astorg et al. ${ }^{(23)}$ & 2008 & France & $\begin{array}{l}276 \text { men, } \\
257 \text { women }\end{array}$ & $\begin{array}{l}\text { Men } 45-60 \text { years, } \\
\text { women } 35-60 \text { years }\end{array}$ & $15 \mathrm{~d} 24 \mathrm{~h}$ records & $\begin{array}{l}\text { Plasma fatty } \\
\text { acids }\end{array}$ & $\begin{array}{l}\text { Crude (men/women): ALA: } 0.06 / 0.05 \dagger \\
\text { EPA: } 0 \cdot 24 / 0 \cdot 27 \dagger^{* * *} \\
\text { DPA: } 0 \cdot 08 / 0.07 \dagger \\
\text { DHA: } 0.25 / 0.27 \dagger^{* \star *}\end{array}$ \\
\hline Sublette et al. ${ }^{(6)}$ & 2010 & USA & 61 & $18-73$ years & $\mathrm{FFQ}$ & $\begin{array}{l}\text { Plasma fatty } \\
\text { acids }\end{array}$ & $\begin{array}{l}\text { Crude: ALA: } 0.22 \dagger \\
\text { EPA: } 0.38 \dagger^{\star \star \star \star} \\
\text { DHA: } 0.50 \dagger,{ }^{* \star \star \star}\end{array}$ \\
\hline
\end{tabular}

DHQ, diet history questionnaire; ALA, $\alpha$-linolenic acid; DPA, docosapentanoic acid.

Significance: ${ }^{*} P<0.05 ;{ }^{* *} P<0.01 ;{ }^{* * *} P<0.001 ;{ }^{* \star * *} P<0.0001$.

† Spearman correlation.

$\ddagger$ Marine intake of $n-3 \mathrm{FA}$

$\S$ Pearson correlation.

II Deattenuated with the within-to-between person variance ratio for intake of FA.

1) Corrected after attenuation correction factor.

††Corrected for the reliability coefficients of FFQ and phospholipids.

$\S \S$ Adjusted for age at blood drawing, BMl, current weight, smoking status, postmenopausal status, postmenopausal hormone use, period of blood assay, and fasting status at blood drawing

|||| Adjusted for age and total energy intake. 
Table 2. Basic characteristics of the included studies on recovery interventions for $n-3$ PUFA biomarkers ${ }^{1}$

\begin{tabular}{|c|c|c|c|c|c|c|c|c|c|c|}
\hline Author/year & Country & Age & Sex & $\begin{array}{l}\text { No. } \\
\text { included }\end{array}$ & $\begin{array}{l}\text { Short description } \\
\text { of intervention }\end{array}$ & $\begin{array}{l}\text { Latest } \\
\text { time } \\
\text { point }\end{array}$ & $\begin{array}{l}\text { No. in } \\
\text { intervention }\end{array}$ & $\begin{array}{l}\text { No. in control } \\
\text { at latest time }\end{array}$ & $\begin{array}{l}\text { Study } \\
\text { design }\end{array}$ & $\begin{array}{l}\text { Biomarkers } \\
\text { reported }\end{array}$ \\
\hline Allard $(1997)^{(37)}$ & Canada & $20-60 y$ & M & 40 & $3.06 \mathrm{~g} \mathrm{EPA}+2.26 \mathrm{~g} \mathrm{DHA}$ & $6 \mathrm{wk}$ & 18 & 19 & RCT $p$ & PPL \\
\hline Barceló-Coblijn (2008) ${ }^{(38)}$ & Canada & $>48$ & $\mathrm{x}$ & 62 & $\begin{array}{l}1.2 \mathrm{~g} \mathrm{ALA} ; 2.4 \mathrm{~g} \text { ALA } ; 3.6 \mathrm{~g} \\
\text { ALA; } 0.6 \mathrm{~g} \text { (fish oil } \\
\text { EPA-DHA), } 1,2 \mathrm{~g} \text { (fish oil } \\
\text { EPA-DHA), } 0.6 \mathrm{~g} / \mathrm{d} \text { EPA or } \\
\text { DHA }+1.2 \mathrm{~g} / \mathrm{d} \text { ALA }\end{array}$ & $12 \mathrm{wk}$ & $\begin{array}{l}12+10+ \\
10+11+ \\
10\end{array}$ & 9 & RCT $p$ & RBC, PPL \\
\hline Bønaa (1992) ${ }^{(39)}$ & Norway & $34-60 y$ & $\mathrm{x}$ & 156 & $3.3 \mathrm{~g} \mathrm{EPA}+1.8 \mathrm{DHA}$ & $10 w k$ & 72 & 74 & RCT p & PPL \\
\hline Brady (2004) $)^{(40)}$ & United Kingdom & $35-70 y$ & M & 29 & $1.47 \mathrm{~g} \mathrm{EPA}+1 \mathrm{~g} \mathrm{DHA}$ & $6 \mathrm{wk}$ & $15+14$ & $15+14$ & $B / A$ & Plat \\
\hline Christensen (1999) ${ }^{(41)}$ & Denmark & $38 \pm 11 y$ & $x$ & 60 & $\begin{array}{l}3 \mathrm{~g} \mathrm{EPA}+2.9 \mathrm{~g} \mathrm{DHA} ; 0.9 \mathrm{~g} \\
\mathrm{EPA}+0.8 \mathrm{~g} \mathrm{DHA}\end{array}$ & $12 \mathrm{wk}$ & $20+20$ & 20 & RCT $p$ & G, Plat \\
\hline Cleland (1992) ${ }^{(42)}$ & Australia & Adults & M & 32 & $1.6 \mathrm{~g} \mathrm{EPA}+0.32 \mathrm{~g} \mathrm{DHA}$ & $4 \mathrm{wk}$ & $13+15$ & $13+15$ & $\mathrm{~B} / \mathrm{A}$ & NPL \\
\hline Conquer $(1998)^{(43)}$ & Canada & $30-34 y$ & $\mathrm{x}$ & 22 & $0.75 \mathrm{~g} \mathrm{DHA} ; 1.5 \mathrm{~g} \mathrm{DHA}$ & $6 \mathrm{wk}$ & $6+7$ & 6 & RCT p & PPL, NEFA \\
\hline Conquer (1999) $)^{(44)}$ & Canada & $29.5 \pm 1.5 y$ & M & 20 & $1.3 \mathrm{~g} \mathrm{EPA}+1.7 \mathrm{~g} \mathrm{DHA}$ & $6 \mathrm{wk}$ & 9 & 10 & RCT p & PPL, NEFA \\
\hline Damsgaard (2007) ${ }^{(45)}$ & Denmark & $9 \mathrm{mo}$ & $\mathrm{x}$ & 94 & $0.57 \mathrm{~g}$ EPA $+0.38 \mathrm{~g} \mathrm{DHA}$ & $12 \mathrm{wk}$ & 24 & 24 & RCT p & $\mathrm{E}$ \\
\hline DeLany $(1990)^{(46)}$ & USA & $19-31$ y & M & 15 & $\begin{array}{l}1.13 \mathrm{~g} \mathrm{EPA}+0.7 \mathrm{~g} \mathrm{DHA} \\
4.53 \mathrm{~g} \mathrm{EPA}+2.73 \mathrm{~g} \mathrm{DHA}\end{array}$ & $5 \mathrm{wk}$ & $5+4$ & 5 & RCT $p$ & PPL \\
\hline Dunstan (2004) ${ }^{(47)}$ & Australia & $32.4 \pm 0.5 y$ & $\mathrm{~F}$ & 98 & $1.1 \mathrm{~g} \mathrm{EPA}+2.2 \mathrm{~g} \mathrm{DHA}$ & $17 \mathrm{wk}$ & 36 & 37 & RCT p & $\mathrm{E}$ \\
\hline Dyerberg $(2004)^{(48)}$ & Denmark & $20-60 y$ & M & 58 & $0.79 \mathrm{~g}$ EPA $+0.5 \mathrm{~g}$ DHA & $8 \mathrm{wk}$ & 24 & 26 & RCT p & Plat \\
\hline Engström (2003) ${ }^{(49)}$ & Sweden & $26-65 y$ & $x$ & 16 & $\begin{array}{l}0.11 \mathrm{~g} \text { EPA + } 0.18 \mathrm{~g} \mathrm{DHA} \\
0.45 \mathrm{~g} \mathrm{EPA}+0.39 \mathrm{~g} \mathrm{DHA}\end{array}$ & $3 w k$ & $8+8$ & $8+8$ & $\mathrm{~B} / \mathrm{A}$ & PPL \\
\hline Hagve (1993) ${ }^{(50)}$ & Norway & $19-22 y$ & $\mathrm{~F}$ & 16 & $3.3 \mathrm{~g}$ EPA $+1.8 \mathrm{~g}$ DHA & $4 \mathrm{wk}$ & 8 & 8 & RCT p & EPL \\
\hline Harris $(2007)^{(51)}$ & USA & $21-49 y$ & $\mathrm{~F}$ & 23 & $0.104 \mathrm{~g} \mathrm{EPA}+0.378 \mathrm{~g} \mathrm{DHA}$ & 16 wk & 11 & 12 & RCT p & RBC, PPL \\
\hline Helland (2006) ${ }^{(52)}$ & Norway & $19-35 y$ & $\mathrm{~F}$ & 341 & $0.8 \mathrm{~g}$ EPA $+1.18 \mathrm{~g} \mathrm{DHA}$ & $17 \mathrm{wk}$ & 158 & 151 & RCT p & PPL \\
\hline Higgins $(2001)^{(53)}$ & Ireland & $19-63 y$ & $x$ & 62 & $\begin{array}{c}0.52 \mathrm{~g} \mathrm{EPA}+0.33 \mathrm{~g} \mathrm{DHA} \\
0.34 \mathrm{~g} \text { EPA }+0.22 \mathrm{~g} \mathrm{DHA} \\
0.17 \mathrm{~g} \text { EPA }+0.11 \mathrm{~g} \mathrm{DHA}\end{array}$ & $16 w k$ & $14+16+17$ & 14 & RCT $p$ & $\mathrm{P}, \mathrm{LDL}$ \\
\hline Hodge $(1993)^{(54)}$ & Australia & $30 \cdot 6 y$ & $\mathrm{~F}$ & 7 & $0.55 \mathrm{~g} \mathrm{EPA}+0.39 \mathrm{~g} \mathrm{DHA}$ & $2 w k$ & 7 & 7 & $\mathrm{~B} / \mathrm{A}$ & $\begin{array}{l}\text { PPL, PCE, PTG, } \\
\text { HDL PL }\end{array}$ \\
\hline Hoffman $(2004)^{(55)}$ & USA & $6 \mathrm{mo}$ & $\mathrm{x}$ & 55 & $0.083 \mathrm{~g} \mathrm{DHA}$ & $26 w k$ & 25 & 26 & RCT p & $\mathrm{E}$ \\
\hline Itomura $(2005)^{(56)}$ & Japan & $9-12 y$ & $\mathrm{x}$ & 179 & $0.12 \mathrm{~g}$ EPA $10.52 \mathrm{~g} \mathrm{DHA}$ & $12 \mathrm{wk}$ & 26 & 23 & RCT p & EPL \\
\hline Katan $(1997)^{(57)}$ & Netherlands & $56 \cdot 2 \pm 16 \cdot 5 y$ & M & 58 & $\begin{array}{c}2.43 \mathrm{~g} \text { EPA + } 0.49 \mathrm{~g} \mathrm{DHA} \\
1.62 \mathrm{~g} \mathrm{EPA}+0.33 \mathrm{~g} \mathrm{DHA} \\
0.81 \mathrm{~g} \mathrm{EPA}+0.16 \mathrm{~g} \mathrm{DHA}\end{array}$ & $52 \mathrm{wk}$ & $14+15+15$ & 14 & RCT $p$ & PCE, E \\
\hline Kew $(2004)^{(58)}$ & United Kingdom & $23-65 y$ & $x$ & 42 & $\begin{array}{c}4.7 \mathrm{~g} \mathrm{EPA}+0.73 \mathrm{~g} \mathrm{DHA} \\
0.85 \mathrm{~g} \text { EPA }+4.9 \mathrm{~g} \mathrm{DHA}\end{array}$ & $4 \mathrm{wk}$ & $11+11$ & 11 & RCT $p$ & PPL, N \\
\hline Khan $(2003)^{(59)}$ & United Kingdom & $40-65 y$ & $\mathrm{x}$ & 56 & $0.02 \mathrm{~g} \mathrm{EPA}+0.94 \mathrm{~g} \mathrm{DHA}$ & $32 \mathrm{wk}$ & 28 & 28 & RCT p & PPL \\
\hline Laidlaw $(2003)^{(60)}$ & Canada & $36-68 y$ & $\mathrm{~F}$ & 8 & $2.32 \mathrm{~g} \mathrm{EPA}+1.68 \mathrm{~g} \mathrm{DHA}$ & $4 \mathrm{wk}$ & 8 & 8 & $\mathrm{~B} / \mathrm{A}$ & PPL \\
\hline Mann $(2010)^{(61)}$ & Australia & $20-50 y$ & $x$ & 27 & $\begin{array}{l}0.21 \mathrm{~g} \text { EPA }+0.03 \mathrm{~g} \\
\text { DPA }+0.81 \mathrm{~g} \mathrm{DHA} ; 0.34 \mathrm{~g} \\
\text { EPA }+0.23 \mathrm{~g} \\
\text { DPA }+0.45 \mathrm{~g} \mathrm{DHA}\end{array}$ & $2 w k$ & $10+8$ & 6 & RCT p & Plat \\
\hline Mantzioris (1994) ${ }^{(62)}$ & Australia & $25-44 y$ & M & 15 & $1.62 \mathrm{~g} \mathrm{EPA}+1.08 \mathrm{~g} \mathrm{DHA}$ & $4 \mathrm{wk}$ & 15 & 15 & $\mathrm{~B} / \mathrm{A}$ & PPL, PCE, PTG \\
\hline McDaniel (2010) ${ }^{(63)}$ & USA & $18-45 y$ & $\mathrm{x}$ & 30 & $1.6 \mathrm{~g} \mathrm{EPA}+1.2 \mathrm{~g} \mathrm{DHA}$ & $4 \mathrm{wk}$ & 16 & 14 & $\mathrm{RCT}$ & PPL \\
\hline Miles $(2004)^{(64)}$ & United Kingdom & $21-44 y$ & M & 50 & $\begin{array}{l}2.1 \mathrm{~g} \text { EPA + 0.9 g DHA; } \\
1.1 \mathrm{~g} \mathrm{EPA}+0.5 \mathrm{~g} \mathrm{DHA} ; \\
0.8 \mathrm{~g} \text { EPA + 0.3 g DHA; } \\
0.6 \mathrm{~g} \text { EPA + 0.2 g DHA }\end{array}$ & $12 \mathrm{wk}$ & $\begin{array}{c}10+10+1- \\
0+10\end{array}$ & 10 & RCT p & $\begin{array}{l}\text { PPL, PCE, PTG, } \\
\text { PBMC }\end{array}$ \\
\hline Mills (1995) ${ }^{(65)}$ & Canada & $21-41 y$ & $x$ & 18 & $0.74 \mathrm{~g} \mathrm{EPA}+0.51 \mathrm{~g} \mathrm{DHA}$ & $6 \mathrm{wk}$ & 8 & 9 & RCT p & E ghosts \\
\hline
\end{tabular}




\begin{tabular}{|c|c|c|c|c|c|c|c|c|c|c|}
\hline Author/year & Country & Age & Sex & $\begin{array}{l}\text { No. } \\
\text { included }\end{array}$ & $\begin{array}{l}\text { Short description } \\
\text { of intervention }\end{array}$ & $\begin{array}{l}\text { Latest } \\
\text { time } \\
\text { point }\end{array}$ & $\begin{array}{l}\text { No. in } \\
\text { intervention }\end{array}$ & $\begin{array}{l}\text { No. in control } \\
\text { at latest time }\end{array}$ & $\begin{array}{l}\text { Study } \\
\text { design }\end{array}$ & $\begin{array}{l}\text { Biomarkers } \\
\text { reported }\end{array}$ \\
\hline Milte $(2007)^{(66)}$ & Australia & $53 y$ & $x$ & 75 & $\begin{array}{c}0,52 \mathrm{~g} \mathrm{DHA}+0,12 \mathrm{~g} \text { EPA; } \\
\text { 1,04 g DHA + 0,24 g EPA; } \\
\text { 1,56 g DHA + 0,36 g EPA }\end{array}$ & $12 \mathrm{wk}$ & $17+20+19$ & 19 & RCT $p$ & $\mathrm{RBC}$ \\
\hline Minns $(2010)^{(67)}$ & USA & $18-36 \mathrm{mo}$ & $x$ & 86 & $0.043 \mathrm{~g} \mathrm{DHA} ; 0.13 \mathrm{~g} \mathrm{DHA}$ & $9 w k$ & $29+29$ & 28 & RCT p & RBC, PPL \\
\hline Montgomery $(2003)^{(68)}$ & United Kingdom & Pregnant & $\mathrm{F}$ & 100 & $0.04 \mathrm{~g} \mathrm{EPA}+0.2 \mathrm{~g} \mathrm{DHA}$ & $25 \mathrm{wk}$ & 30 & 29 & RCT p & $E, P$ \\
\hline Murphy $(2007)^{(69)}$ & Australia & $20-65 y$ & $x$ & 86 & $0.125 \mathrm{~g} \mathrm{EPA}+\mathrm{DHA}$ & $24 w k$ & 38 & 32 & RCT p & $\mathrm{RBC}$ \\
\hline Neubronner $(2011)^{(70)}$ & Germany & $30-75 y$ & $x$ & 129 & $1.01 \mathrm{~g} \mathrm{EPA}+0,67 \mathrm{~g} \mathrm{DHA}$ & $24 \mathrm{wk}$ & $41+45$ & 43 & RCT p & $\mathrm{RBC}$ \\
\hline Otto $(2000)^{(71)}$ & Netherlands & $20-45 y$ & $\mathrm{~F}$ & 75 & $\begin{array}{c}0.06 \mathrm{~g} \text { EPA }+0.27 \mathrm{~g} \mathrm{DHA} \\
0.12 \mathrm{~g} \mathrm{EPA}+0.53 \mathrm{~g} \mathrm{DHA} ; \\
0.29 \mathrm{~g} \mathrm{DHA} ; 0.57 \mathrm{~g} \mathrm{DHA}\end{array}$ & $4 \mathrm{wk}$ & $\begin{array}{c}15+15+1- \\
4+12\end{array}$ & 15 & RCT p & PPL, EPL \\
\hline Otto $(2000)^{(72)}$ & Netherlands & $20-38 y$ & $\mathrm{~F}$ & 24 & $0.57 \mathrm{~g} \mathrm{DHA}$ & 4 wk & 12 & 12 & RCT p & PPL, EPL \\
\hline Palozza (1996) ${ }^{(73)}$ & Italy & $25-46 y$ & $x$ & 40 & $\begin{array}{l}4.1 \mathrm{~g} \text { EPA + } 3.6 \mathrm{~g} \mathrm{DHA} ; \\
2.7 \mathrm{~g} \mathrm{EPA}+2.4 \mathrm{~g} \mathrm{DHA} ; \\
1.4 \mathrm{~g} \text { EPA }+1.1 \mathrm{~g} \mathrm{DHA}\end{array}$ & $26 \mathrm{wk}$ & $10+10+10$ & 10 & RCT $p$ & $P, E$ \\
\hline Park $(2002)^{(74)}$ & USA & $37-43 y$ & $x$ & 33 & $4 \mathrm{~g} \mathrm{EPA}$ or $4 \mathrm{~g} \mathrm{DHA}$ & $4 \mathrm{wk}$ & $10+10$ & 11 & RCT p & Plat \\
\hline Rees $(2006)^{(75)}$ & United Kingdom & $18-70 y$ & $M$ & 169 & $\begin{array}{l}1.35 \mathrm{~g} \mathrm{EPA}+0.3 \mathrm{~g} \mathrm{DHA} \\
2.7 \mathrm{~g} \mathrm{EPA}+0.6 \mathrm{~g} \mathrm{DHA} ; \\
4.5 \mathrm{~g} \mathrm{EPA}+0.9 \mathrm{~g} \mathrm{DHA}\end{array}$ & $12 w k$ & $39+38+38$ & 40 & RCT p & PPL, PBMC PL \\
\hline Sanders $(2006)^{(76)}$ & United Kingdom & $29-35 y$ & $x$ & 80 & $1.5 \mathrm{~g} \mathrm{DHA}$ & $4 \mathrm{wk}$ & 40 & 39 & RCT p & $P, E P L$ \\
\hline Sanjurjo $(2004)^{(77)}$ & Spain & $31-34 y$ & $\mathrm{~F}$ & 20 & $0.04 \mathrm{~g} \mathrm{EPA}+0.2 \mathrm{~g} \mathrm{DHA}$ & $14 \mathrm{wk}$ & 8 & 8 & RCT p & $\mathrm{P}$ \\
\hline Smuts $(2003)^{(78)}$ & USA & $16-35 y$ & $\mathrm{~F}$ & 48 & $0.184 \mathrm{~g} \mathrm{DHA}$ & $14 \mathrm{wk}$ & 18 & 19 & RCT p & PPL, PTG, EPL \\
\hline Sørensen $(1998)^{(79)}$ & Denmark & $29-60 y$ & M & 50 & $0.37 \mathrm{~g}$ EPA $+0.54 \mathrm{~g} \mathrm{DHA}$ & $4 \mathrm{wk}$ & 21 & 24 & RCT p & LDL \\
\hline Stark $(2000)^{(80)}$ & Canada & $43-60 y$ & $\mathrm{~F}$ & 36 & $2.4 \mathrm{~g} \mathrm{EPA}+1.6 \mathrm{~g} \mathrm{DHA}$ & $4 \mathrm{wk}$ & 18 & 17 & RCT p & PPL \\
\hline Surai $(2000)^{(81)}$ & United Kingdom & $26-59 y$ & $x$ & 44 & $0.21 \mathrm{~g} \mathrm{DHA}$ & $8 w k$ & 20 & 20 & RCT p & $\begin{array}{l}\text { PPL, PCE, PTG, } \\
\text { NEFA }\end{array}$ \\
\hline Thies $(2001)^{(82)}$ & United Kingdom & $56-69 y$ & $x$ & 24 & $\begin{array}{l}0.7 \mathrm{~g} \mathrm{DHA} ; 0.72 \mathrm{~g} \\
\mathrm{EPA}+0.28 \mathrm{~g} \text { DHA }\end{array}$ & $12 \mathrm{wk}$ & $8+7$ & 8 & RCT p & PBMC PL \\
\hline Vognild $(1998)^{(83)}$ & Norway & $16-69 y$ & $x$ & 228 & $\begin{array}{l}0.7 \mathrm{~g} \text { EPA }+1 \mathrm{~g} \mathrm{DHA} ; 0.5 \mathrm{~g} \\
\text { EPA }+0.6 \mathrm{~g} \mathrm{DHA} ; 0.5 \mathrm{~g} \\
\text { EPA }+0.8 \mathrm{~g} \mathrm{DHA} ; 1 \mathrm{~g} \\
\text { EPA }+1.5 \mathrm{~g} \mathrm{DHA} ; 1.3 \mathrm{~g} \\
\text { EPA }+1.8 \mathrm{~g} \mathrm{DHA}\end{array}$ & $12 w k$ & $\begin{array}{l}35+36+3- \\
8+36+3- \\
4\end{array}$ & 36 & RCT p & $\mathrm{P}$, Plat \\
\hline Wallace $(2000)^{(84)}$ & Ireland & $20-26 y$ & $\mathrm{~F}$ & 25 & $0.35 \mathrm{~g} \mathrm{EPA}+0.32 \mathrm{~g} \mathrm{DHA}$ & $4 \mathrm{wk}$ & $13+12$ & $13+12$ & & Plat \\
\hline Yaqoob $(2000)^{(85)}$ & United Kingdom & $39-49 y$ & $x$ & 16 & $2.1 \mathrm{gEPA}+1.1 \mathrm{~g} \mathrm{DHA}$ & $12 \mathrm{wk}$ & 8 & 8 & RCT p & PPL, PBMC \\
\hline
\end{tabular}

$1 \mathrm{M}$, exclusively male group; EPA, eicosapentaenoic acid; DHA, docosahexaenoic acid; RCT, randomized controlled trial; $p$, parallel; PPL, plasma phospholipids; $\mathrm{X}$, mixed sex group; ALA, a-linolenic acid; B/A, before-after study; Plat, total platelets; G, total granulocyte; NPL, neutrophil phospholipid; NEFA, nonesterified fatty acids; E, total erythrocytes; F, exclusively female group; EPL, erythrocyte phospholipids; P, total plasma; PCE, plasma cholesteryl esters; PTG, plasma triacylglycerols; PL, phospholipids; N, total neutrophils; DPA, docosapentanoic acid; PBMC, peripheral blood mononuclear cells 
between dietary intake of fatty acids estimated from the FFQ in controls and concentrations of fatty acid in plasma of 0.38 for EPA and 0.49 for DHA. Godley et al. ${ }^{(10)}$ reported correlations between dietary intake of EPA and DHA estimated from the FFQ and concentrations of EPA and DHA in erythrocyte membrane ranging from $0 \cdot 19$ to $0 \cdot 36$. This is the smallest value found for DHA in the blood biomarkers. Sublette et al. ${ }^{(6)}$ reported significant correlations between dietary intake of ALA, EPA and DHA estimated from the FFQ and concentrations of ALA, EPA and DHA in plasma of 0.22 for ALA, 0.38 for EPA and 0.50 for DHA, which was the highest value found for the different biomarkers utilized. Sun et al. ${ }^{(15)}$ reported a significant adjusted correlation of 0.56 between dietary intake of DHA estimated from the FFQ and concentrations of DHA in erythrocytes.

One dietary history questionnaire was also validated against serum fatty acid and high crude ( $r 0.46)$ and adjusted ( $r 0.59)$ correlations were reported for intake of EPA for men ${ }^{(18)}$. This questionnaire was self-administered and was somewhat similar to a FFQ.

Three studies have validated weighed records (all with seven or more days) against serum, erythrocytes or plasma fatty acids ${ }^{(12,20,21)}$. Kobayashi et al. ${ }^{(20)}$ presented a very high correlation coefficient for EPA, crude $(r$ 0.75) and adjusted ( $r$ 0.89), as well as the best adjusted correlations for DHA and total $n$-3 PUFA from weighed records validated against serum fatty acids ( $r$ 0.61). Kuriki et $a l .{ }^{(21)}$ obtained adjusted correlations for dietary intake of EPA measured with weighed records against plasma concentrations of EPA $(r 0.57)$ and for DHA ( $r$ 0.57). McNaughton et al. ${ }^{(12)}$ showed a crude correlation of 0.43 for DHA measured with weighed records validated against DHA concentration in plasma and a lower correlation coefficient for EPA $(r$ 0.22). Similar correlations were observed when the intake was measured with a FFQ (DHA $r 0.32$ and EPA $r$ 0.21). All three studies presented low correlations for $\operatorname{ALA}^{(12,20,21)}$.

\section{Biomarker study}

A total of 8 new studies were incorporated to the 41 previously included papers identified in the study by Fekete et $a{ }^{(5)}$.

Details of the biomarkers analysed are given in Table 2 wich include 49 recovery studies of $n-3$ PUFA biomarkers. In this study, as summarized in Table 3, Total plasma lipid DHA appears to be a good biomarker of DHA status, which reacts rapidly to supplementation and is sensitive to supplementation dose. It appears to be reliable in adults, mixed sex studies, and those with moderate baseline DHA status, but it is not clear for which other population subgroups its application can be reliable. Moreover, plasma phospholipid DHA appears to be a good biomarker of DHA status. It reacts rapidly to supplementation and is also sensitive to supplementation dose. This biomarker appears to respond appropriately in adults, males, females, those with low, moderate, or high baseline DHA status, those who used marine oil, seafood, or single cell oils, and in those whose dose amounts were $\leq 2500 \mathrm{mg} / \mathrm{d}$ of DHA. There were insufficient studies to assess the effectiveness of plasma phospholipid DHA in other population subgroups. With reference to Plasma phospholipid EPA, it appeared to be a good biomarker for EPA status in men and women and those who had low, moderate,

Table 3. Primary analyses (pooled data on the longest duration and the highest supplementation dose) for each identified biomarker ${ }^{1}$

\begin{tabular}{|c|c|c|c|c|}
\hline Biomarker & $\begin{array}{c}\text { No. of studies } \\
\text { (no. of included participants) }\end{array}$ & $\begin{array}{l}\text { Pooled effect size, WMD } \\
(95 \% \mathrm{Cl})^{2}\end{array}$ & $I^{2}$ & Appears effective as a biomarker? ${ }^{3}$ \\
\hline Plasma DHA & $6(262)$ & $1.13(0.54,1.71)$ & 88.7 & Yes \\
\hline Plasma phospholipid DHA & $24(1023)$ & $2.51(1.97,3.00)$ & 95.0 & Yes \\
\hline Plasma phospholipid EPA & $16(759)$ & $4.07(2.90,5.24)^{4}$ & $99 \cdot 0$ & Yes \\
\hline Plasma triacylglycerol DHA & $5(116)$ & $0.86(0.08,1.65)$ & $92 \cdot 1$ & Yes \\
\hline Plasma cholesteryl ester DHA & $5(110)$ & $0.42(0.13,0.71)$ & $92 \cdot 2$ & Yes \\
\hline Plasma nonesterified DHA & $3(72)$ & $1.35(0.11,2.59)$ & $95 \cdot 0$ & Yes \\
\hline Erythrocyte DHA & $12(570)$ & $2.43(1,04,3.91)$ & $95 \cdot 0$ & Yes \\
\hline Erythrocyte phospholipid DHA & $6(229)$ & $0.97(0.50,1.43)$ & $72 \cdot 3$ & Yes \\
\hline Young erythrocyte ghosts DHA & $1(17)$ & $-1.00(-4.07,2.07)^{5}$ & $\mathrm{~N} / \mathrm{A}$ & Unclear \\
\hline Old erythrocyte ghosts DHA & $1(17)$ & $1.70(0.32,3.08)^{5}$ & $N / A$ & Unclear \\
\hline Platelet DHA & $9(251)$ & $1.29(0.92,1.56)$ & 81.9 & Yes \\
\hline Granulocyte DHA & $1(40)$ & $0.60(0.32,0.88)$ & $N / A$ & Unclear \\
\hline Neutrophil DHA & $1(20)$ & $2.80(0.01,5.59)$ & $N / A$ & Unclear \\
\hline Neutrophil phospholipid DHA & $2(28)$ & $0.04(-0.15,0.23)$ & $N / A$ & Unclear \\
\hline PBMC DHA & $2(36)$ & $0.06(-0.36,0.48)$ & 0 & Unclear \\
\hline PBMC phospholipid DHA & $3(94)$ & $0.70(-0.66,2.06)$ & 93.9 & Unclear \\
\hline LDL DHA & $2(73)$ & $0.60(0.59,0.61)$ & 0 & Unclear \\
\hline HDL phospholipid DHA & $1(7)$ & $0.80(0.07,1.53)$ & $N / A$ & Unclear \\
\hline
\end{tabular}

Modified and updated from Fekete et al. ${ }^{(5)}$

1 WMD, weighted mean difference; DHA, docosahexaenoic acid; EPA, eicosapentaenoic acid; N/A, no available data; PBMC, peripheral blood mononuclear cell.

2 All studies are in \%DHA of total fatty acids unless otherwise stated.

3 To claim that a biomarker was effective (reflected change in status) within a review, 3 conditions needed to be met: (1) statistical significance within a forest plot ( $95 \%$ Cl did not include 0 or $P<0.05$ ); (2) $\geq 3$ trials contributed data; and (3) between intervention and control arms in the studies contributing data there were $\geq 50$ participants. To claim that a biomarker was ineffective, 4 conditions had to be met: (1) lack of statistical significance within a forest plot (95\% $\mathrm{Cl}$ included 0 or $P \geq 0.05$ ); (2) $\geq 3$ trials contributed data; (3) between intervention and control arms in the studies contributing data there were $\geq 50$ participants; and (4) study results were approximately similar (heterogeneity levels were acceptable so that $I^{2}<50 \%$ ).

$4 \%$ EPA of total fatty acid.

$5 \mu \mathrm{g} / \mathrm{mg}$ protein. 
or high baseline EPA, which reacts rapidly to supplementation and is sensitive to supplementation dose.

Plasma triacylglycerol DHA could be a good biomarker of DHA status, but there were insufficient studies to allow exploration of which population groups it may be most effective in. Plasma cholesteryl ester DHA appears to be a good biomarker of DHA status at lower-dose supplementation, but it is not clear within which population groups it is effective or whether it works well at higher doses of supplementation. Plasma nonesterified fatty acid DHA may be a good biomarker of DHA status, but there were insufficient studies to allow for the exploration of appropriateness of its use in different population groups and doses. Erythrocyte membrane total lipid DH appears to be a good biomarker of DHA status, and the data suggest that there is a dose response. Although it seems to be an effective biomarker in infants for most doses, confirmation is not possible due to limited data. Erythrocyte membrane phospholipid DHA appears to be a good biomarker of DHA status and although it seems to be an effective biomarker in adults, children and adolescents, as well as in pregnant or lactating women and at most doses, this cannot be confirmed due to limited data. Total platelet lipid DHA could be a good biomarker of DHA status, but there was no apparent dose response. For Peripheral blood mononuclear cell phospholipid DHA, the response of peripheral blood mononuclear cell phospholipid DHA values to DHA supplementation did not appear to be a good biomarker of DHA status. For other potential biomarkers of DHA status, evidence was insufficient for young erythrocyte ghost DHA, old erythrocyte ghost DHA, granulocyte DHA, neutrophil DHA, neutrophil phospholipid DHA, peripheral blood mononuclear cell total lipid DHA, LDL DHA, and HDL phospholipid DHA.

\section{Discussion}

In a validation study, the reference method used should be as accurate as possible ${ }^{(8)}$. A validation study is also called a relative validation/calibration study when one dietary method is compared to another dietary method, most often FFQ $v$. several days of food records. The limitations with this approach are the considerable individual day-to-day variation, which reduces the possibility of obtaining a true measure of usual intake with few recording days, as well as reporting bias since both self-administered dietary assessment questionnaires and dietary records are based on self-reporting ${ }^{(8)}$. FFQs often overestimate intake of energy and nutrients, while food records often underreport energy and nutrient intakes ${ }^{(24,25)}$. As such, we thought it best to exclude those questionnaires where validation was made against another dietary measurement tool. An alternative to relative validations is the use of biomarkers, whose primary advantage is that these measurements are objective and the sources of errors for a biomarker and a dietary assessment method are independent ${ }^{(8,26)}$. The $n-3$ PUFA are largely exogenic, meaning that there is no synthesis of $n$ - 3 PUFA in the body and that intake via diet and supplements are the major source, making the correlations with biomarkers easier ${ }^{(11,27)}$. There are several choices of a biomarker for the measurement of LC $n-3$ PUFA, and those presented in this review were fatty acids in adipose tissue, erythrocytes and plasma. Adipose tissue fatty acids are generally considered the best source of assessing longterm fatty acid intake ${ }^{(13,27)}$. Erythrocytes may be a useful marker as they can provide an indication of the previous 120-d intake of LC $n-3$ PUFA $^{(1)}$. Plasma fatty acids reflect intake of fatty acids over the past few days or more ${ }^{(11)}$. Most of the included studies have presented the correlations, both crude and adjusted. The correlation coefficients obtained from the validation studies can reflect the capability of the method to rank individuals according to fatty acid intake.

\section{Subcutaneous fat}

Fatty acids estimated from six different $\mathrm{FFQ}^{(7,8,10,13,14)}$, one weighed record $^{(19)}$ and one recall ${ }^{(13)}$ were validated against subcutaneous fat, which the literature describes as the best reference method. The correlation coefficients observed in all the studies were in the range of 0.40-0.66 for ALA, EPA and DHA. In summary, none of the dietary methods validated against subcutaneous fat and presented here seem to be superior than the others in relation to ranking the dietary intake of $n-3$ PUFA. Two articles related to subcutaneous fat were found for this updated review, the correlation coefficient range for EPA was $0 \cdot 15-0.33$ and for DHA $0 \cdot 18-0 \cdot 42$. This suggests a weaker correlation when compared to the previous correlation reported by Marckman et al. ${ }^{(19)}$ which showed the highest correlation coefficient (0.66) amongst all the included studies.

\section{Blood component composition}

Dietary intake of $n$-3 PUFA estimated from eleven different $\mathrm{FFQ}^{(1,6,8-12,15-17,22)}$, one diet history questionnaire ${ }^{(18)}$ and three weighed record studies ${ }^{(12,20,21)}$ was validated against fatty acids in serum, plasma or erythrocytes. Both fatty acids in plasma, erythrocytes and serum were found to be good biomarkers of LC $n-3$ PUFA $^{(1,22)}$. The correlation coefficients observed between the intake of fatty acids measured by most FFQs ${ }^{(1,7,8)}$, the diet history questionnaire ${ }^{(1)}$, and the weighed records ${ }^{(21)} v$. fatty acid in blood parameters were at the same range $(r$ 0.40-0.60). The best correlation was observed in the study by Kobayashi et al. ${ }^{(20)}$ comparing the dietary intake of fatty acids from weighed records with fatty acids in serum phospholipids (EPA, $r$ 0.89). However, there was no clear tendency among the three studies comparing fatty acids from weighed records with fatty acids in $\operatorname{blood}^{(12,20,21)}$. As such, it seems that weighed records were the best method to measure $n$ - 3 PUFA intake.

Most correlation coefficients from the studies comparing dietary intake with fatty acids in blood parameters were in the same range as the ones observed for fatty acids in adipose tissue $(r 0 \cdot 40-0 \cdot 60)$. There were two studies with a lower correlation $^{(12,23)}$ and one with a correlation higher than this range ${ }^{(20)}$. For ALA most studies presented low correlations between dietary intake and blood parameters in both previous and updated versions of this review ${ }^{(6,15,17,23)}$. In the present updated review, the correlation coefficient range for 
erythrocyte EPA was $0 \cdot 23-0 \cdot 38$ and for erythrocyte DHA 0.190.56 which suggests an acceptable and a reasonable good correlation coefficient respectively ${ }^{(10,15)}$. Regarding plasma phospholipid EPA the correlation range was $0 \cdot 21-0 \cdot 38$ and for DHA $0 \cdot 25-0 \cdot 50^{(6,15,23)}$, again showing an acceptable and reasonably good correlation coefficient, respectively. It is important to highlight that none of the current correlation coefficients found in this updated review were higher than those previously reported in the original article; however the same levels were maintained. This implies that any additional validation study will unlikely produce higher correlation estimates between questionnaires and biomarkers.

The estimation of summarised crude and adjusted correlations for all the validation studies of FFQs using biomarkers as the reference method indicates that the FFQ gives 'acceptable' values for total $n$ - 3 PUFA, EPA and DHA. The summarised crude and adjusted correlations for the two studies validating weighed records against biomarkers indicate 'acceptable' estimates for total $n$-3 PUFA, while the estimates obtained a higher ranking of 'good' for EPA and DHA. As expected, the weighed records seem to be superior to the FFQ in reference to estimating intakes of EPA and DHA.

Biomarkers were more accurate than different dietary methods to rank individuals. One limitation with food records is that subjects are prone to underestimate their food intake when they keep food records ${ }^{(24)}$. The true food consumption of $n-3$ FA most likely lies somewhere between the weighed records and the FFQ. According to the systematic review, none of the dietary assessment methods used to assess $n-3$ PUFA seem to be highly superior to another, with weighed records being slightly better than FFQs. Most studies presented correlation coefficients ranging from 0.40 to 0.60 . This review also confirmed the view that employing an FFQ to assess $n$-3 PUFA requires that it be validated against reliable and valid biomarkers, and that validation studies of dietary methods for measuring intakes of $n-3$ FA could be improved.

Additionally, after analyzing the 18 different potential biomarkers of LC $n-3$ PUFA status reported in Table 3 , we could argue that plasma phospholipid DHA, erythrocyte DHA and platelet DHA were all effective biomarkers of DHA status. With regard to other biomarkers (plasma DHA, plasma triacylglycerol DHA, plasma cholesteryl ester DHA, plasma nonesterified DHA, erythrocyte phospholipid DHA, and plasma phospholipid EPA) we could not find any additional evidence to support modifying or promoting previously published statements from Fekete et al. ${ }^{(5)}$.

Still and all, it is worth noting that the health benefits of increasing LC $n$-3 PUFA dietary intake need to be evaluated in RCTs investigating specific clinical outcomes. There are some clear limitations found. First, the number of studies reporting data on different potential biomarkers is limited. This situation reduced our ability to explore which population subgroups or in which types of intervention the biomarkers are effective. Second, we were able to focus on the effect of supplementing DHA only, whereas LC $n$-3 PUFA supplementation usually consists of a complex mixture of LC $n-3$ PUFAs that may interconvert with each other ${ }^{(27)}$. Third, the dose-response curve of the incorporation of DHA (or any other fatty acid) may differ between the distinct blood component constituents ${ }^{(28,29)}$; hence, it may be assumed, with good reason, that the uniform time duration and dose categories may have differently influenced the evaluation of the biomarkers.

Although several clinical studies have investigated the response of various biomarkers to modified $n-3$ fatty acid intake ${ }^{(28-33)}$ and important theoretical considerations have also been published ${ }^{(34,35,36)}$, we still do not have enough data available in the literature. Which biomarker might be sensitive enough to detect changes of a given dose of LC $n-3$ PUFA supplementation in a given clinical condition or population group? Further research is needed to characterize and to understand the meaning of the different correlations between intake estimates and biomarkers of LC n3 PUFA in distinct population groups and environments.

\section{Acknowledgements and disclosures}

The preparatory meetings for this series of reviews on fat and health were funded by Puleva Food. Neither Lluis Serra-Majem nor Mariela Nissensohn, Nina C Øverby or Katalin Fekete have conflicts of interest to disclose. Lluis Serra-Majem and Mariela Nissensohn contributed to the design of the strategy for the literature search, double screened and selected the retrieved documents. Authors acknowledge Alexandra Santos Johnson and Melania Morales Sanchez from de Department of Clinical Sciences of the University of Las Palmas de Gran Canaria (ULPGC) the support provided to select and retrieve the documents, as well as Francisco Fumagallo from the Library of the School of Health Sciences at the ULPGC. Nina C Øverby and Katalin Fekete provided previous literature searches and analysis. Lluis Serra-Majem prepared the main outline of the manuscript and all authors contributed to the preparation of the manuscript.

\section{References}

1. Sullivan BL, Williams PG \& Meyer BJ (2006) Biomarker validation of a long-chain omega-3 polyunsaturated fatty acid food frequency questionnaire. Lipids 41, 845-850.

2. Ashwell M, Lambert JP, Alles MS, et al. (2008) How we will produce the evidence-based EURRECA toolkit to support nutrition and food policy. Eur J Nutr 47, Suppl. 1, 2-16.

3. Øverby N, Serra-Majem L \& Frost Andersen L (2009) Dietary assessment methods on $n-3$ fatty acid intake: a systematic review. Br J Nutr 102, S56-S63.

4. Serra-Majem L, Frost Andersen L, Henriquez-Sánchez P, et al. (2009) Evaluating the quality of dietary intake validation studies. Br J Nutr 102, Suppl. 1, S3-S9.

5. Fekete K, Marosvölgyi T, Jakobik V, et al. (2009) Methods of assessment of $n-3$ long-chain polyunsaturated fatty acid status in humans: a systematic review. Am J Clin Nutr 89, 2070S-2084S.

6. Sublette ME, Segal-Isaacson CJ, Cooper TB, et al. (2011) Validation of a food frequency questionnaire to assess intake of $n-3$ polyunsaturated fatty acids in subjects with and without major depressive disorder. J Am Diet Assoc 111, 117-123.

7. Hunter DJ, Rimm EB, Sacks FM, et al. (1992) Comparison of measures of fatty acid intake by subcutaneous fat aspirate, 
food frequency questionnaire, and diet records in a freeliving population of US men. Am J Epidemiol 135, 418-427.

8. Andersen LF, Solvoll K, Johansson LR, et al. (1999) Evaluation of a food frequency questionnaire with weighed records, fatty acids, and alpha-tocopherol in adipose tissue and serum. Am J Epidemiol 150, 75-87.

9. Arsenault LN, Matthan N, Scott TM, et al. (2009) Validity of estimated dietary eicosapentaenoic acid and docosahexaenoic acid intakes determined by interviewer-administered food frequency questionnaire among older adults with mild-to-moderate cognitive impairment or dementia. Am J Epidemiol 170, 95-103.

10. Godley PA, Campbell MK, Miller C, et al. (1996) Correlation between biomarkers of omega-3 fatty acid consumption and questionnaire data in African American and Caucasian United States males with and without prostatic carcinoma. Cancer Epidemiol Biomarkers Prev 5, 115-119.

11. Hodge AM, Simpson JA, Gibson RA, et al. (2007) Plasma phospholipid fatty acid composition as a biomarker of habitual dietary fat intake in an ethnically diverse cohort. Nutr Metab Cardiovasc Dis 17, 415-426.

12. McNaughton SA, Hughes MC \& Marks GC (2007) Validation of a FFQ to estimate the intake of PUFA using plasma phospholipid fatty acids and weighed foods records. Br J Nutr $\mathbf{9 7}$, 561-568

13. Knutsen SF, Fraser GE, Beeson WL, et al. (2003) Comparison of adipose tissue fatty acids with dietary fatty acids as measured by 24-hour recall and food frequency questionnaire in Black and White Adventists: the Adventist Health Study. Ann Epidemiol 13, 119-127.

14. Baylin A, Kabagambe EK, Siles X, et al. (2002) Adipose tissue biomarkers of fatty acid intake. Am J Clin Nutr 76, 750-757.

15. Sun Q, Ma J, Campos H, et al. (2007) Comparison between plasma and erythrocyte fatty acid content as biomarkers of fatty acid intake in US women. Am J Clin Nutr 86, 74-81.

16. Norrish AE, Skeaff CM, Arribas GL, et al. (1999) Prostate cancer risk and consumption of fish oils: a dietary biomarker-based case-control study. $\mathrm{Br} J$ Cancer $\mathbf{8 1}$, $1238-1242$.

17. Ma J, Folsom AR, Shahar E, et al. (1995) Plasma fatty acid composition as an indicator of habitual dietary fat intake in middle-aged adults. The Athero-sclerosis Risk in Communities (ARIC) Study Investigators. Am J Clin Nutr 62, 564-571.

18. Sasaki S, Ushio F, Amano K, et al. (2000) Serum biomarkerbased validation of a self administered diet history questionnaire for Japanese subjects. J Nutr Sci Vitaminol.(Tokyo) 46 , 285-296.

19. Marckmann P, Lassen A, Haraldsdottir J, et al. (1995) Biomarkers of habitual fish intake in adipose tissue. Am J Clin Nutr 62, 956-959.

20. Kobayashi M, Sasaki S, Kawabata T, et al. (2001) Single measurement of serum phospholipid fatty acid as a biomarker of specific fatty acid intake in middle-aged Japanese men. Eur J Clin Nutr 55, 643-650.

21. Kuriki K, Nagaya T, Tokudome Y, et al. (2003) Plasma concentrations of ( $n-3)$ highly unsaturated fatty acids are good biomarkers of relative dietary fatty acid intakes: a crosssectional study. J Nutr 133, 3643-3650.

22. Hjartaker A, Lund E \& Bjerve KS (1997) Serum phospholipid fatty acid composition and habitual intake of marine foods registered by a semi-quantitative food frequency questionnaire. Eur J Clin Nutr 51, 736-742.

23. Astorg P, Bertrais S, Laporte F, et al. (2008) Plasma n-6 and $n-3$ polyunsaturated fatty acids as biomarkers of their dietary intakes: a cross-sectional study within a cohort of middle-aged French men and women. Eur J Clin Nutr 62 , $1155-1161$.

24. Livingstone MB, Prentice AM, Strain JJ, et al. (1990) Accuracy of weighed dietary records in studies of diet and health. BMJ 300, 708-712.

25. Black AE, Goldberg GR, Jebb SA, et al. (1991) Critical evaluation of energy intake data using fundamental principles of energy physiology: 2. Evaluating the results of published surveys. Eur J Clin Nutr 45, 583-599.

26. Arab L (2003) Biomarkers of fat and fatty acid intake. J. Nutr 133, Suppl. 3, 925S-932S.

27. Arterburn LM, Hall EB \& Oken H (2006) Distribution, interconversion, and dose response of $n-3$ fatty acids in humans. Am J Clin Nutr 83, 1467S-1476S.

28. Brown AJ, Pang E \& Roberts DC (1991) Persistent changes in the fatty acid composition of erythrocyte membranes after moderate intake of $n-3$ polyunsaturated fatty acids: study design implications. Am J Clin Nutr 54, 668-673.

29. Tremoli E, Maderna P, Marangoni F, et al. (1995) Prolonged inhibition of platelet aggregation after $n 2$ fatty acid ethyl ester ingestion by healthy volunteers. Am J Clin Nutr 61, 607-613.

30. Silverman DI, Ware JA, Sacks FM, et al. (1991) Comparison of the absorption and effect on platelet function of a single dose of $n-3$ fatty acids given as fish or fish oil. Am J Clin Nutr 53, 1165-1170.

31. Nordøy A, Barstad L, Connor WE, et al. (1991) Absorption of the $n-3$ eicosapentaenoic and docosahexaenoic acids as ethyl esters and triglycerides by humans. Am J Clin Nutr 53, 1185-1190.

32. Zuijdgeest-van Leeuwen SD, Dagnelie PC, Rietveld T, et al. (1999) Incorporation and washout of orally administered $n-3$ fatty acid ethyl esters in different plasma lipid fractions. Br J Nutr 82, 481-488.

33. Cao J, Schwichtenberg KA, Hanson NQ, et al. (2006) Incorporation and clearance of omega-3 fatty acids in erythrocyte membranes and plasma phospholipids. Clin Chem 52, $2265-2272$.

34. Harris WS, Sands SA, Windsor SL, et al. (2004) Omega-3 fatty acids in cardiac biopsies from heart transplantation patients: correlation with erythrocytes and response to supplementation. Circulation 110, 1645-1649.

35. Fokkema MR, Smit EN, Martini IA, et al. (2002) Assessment of essential fatty acid and omega3-fatty acid status by measurement of erythrocyte 20:3 omega 9 (Mead acid), 22:5 omega 6/20: 4 omega 6 and 22:5 omega 6/22:6 omega3. Prostaglandins Leukot Essent Fatty Acids 67, 345-356.

36. Harris WS, Assaad B \& Poston WC (2006) Tissue omega-6/ omega-3 fatty acid ratio and risk for coronary artery disease. Am J Cardiol 98, 19i-26i.

37. Allard JP, Kurian R, Aghdassi E, et al. (1997) Lipid peroxidation during $n-3$ fatty acid and vitamin E supplementation in humans. Lipids 32, 535-541.

38. Barceló-Coblijn G, Murphy E, Othman R, et al. (2008) Flaxseed oil and fish-oil capsule consumption alters human red blood cell $n$-3 fatty acid composition: a multiple-dosing trial comparing 2 sources of $n$-3 fatty acid. Am J Clin Nutr 88, 801-809.

39. Bønaa KH, Bjerve KS \& Nordøy A (1992) Docosahexaenoic and eicosapentaenoic acids in plasma phospholipids are divergently associated with high density lipoprotein in humans. Arterioscler Thromb 12, 675-681.

40. Brady LM, Lovegrove SS, Lesauvage SV, et al. (2004) Increased $n 26$ polyunsaturated fatty acids do not attenuate the effects of long-chain $n-3$ polyunsaturated fatty acids on 
insulin sensitivity or triacylglycerol reduction in Indian Asians. Am J Clin Nutr 79, 983-991.

41. Christensen JH, Christensen MS, Dyerberg J, et al. (1999) Heart rate variability and fatty acid content of blood cell membranes: a doseresponse study with $n$-3 fatty acids. $A m$ J Clin Nutr 70, 331-337.

42. Cleland LG, James MJ, Neumann MA, et al. (1992) Linoleate inhibits EPA incorporation from dietary fish-oil supplements in human subjects. Am J Clin Nutr 55, 395-399.

43. Conquer JA \& Holub BJ (1998) Effect of supplementation with different doses of DHA on the levels of circulating DHA as non-esterified fatty acid in subjects of Asian Indian background. J Lipid Res 39, 286-292.

44. Conquer JA, Cheryk LA, Chan E, et al. (1999) Effect of supplementation with dietary seal oil on selected cardiovascular risk factors and hemostatic variables in healthy male subjects. Thromb Res 96, 239-250.

45. Damsgaard CT, Lauritzen L, Kjaer TM, et al. (2007) Fish oil supplementation modulates immune function in healthy infants. J Nutr 137, 1031-1036.

46. DeLany JP, Vivian VM, Snook JT, et al. (1990) Effects of fish oil on serum lipids in men during a controlled feeding trial. Am J Clin Nutr 52, 477-485.

47. Dunstan JA, Mori TA, Barden A, et al. (2004) Effects of $n$-3 polyunsaturated fatty acid supplementation in pregnancy on maternal and fetal erythrocyte fatty acid composition. Eur J Clin Nutr 58, 429-437.

48. Dyerberg J, Eskesen DC, Andersen PW, et al. (2004) Effects of trans- and $n-3$ unsaturated fatty acids on cardiovascular risk markers in healthy males. An 8 weeks dietary intervention study. Eur J Clin Nutr 58, 1062-1070.

49. Engstro K, Wallin R \& Saldeen T (2003) Effects of Scandinavian caviar paste enriched with a stable fish oil on plasma phospholipid fatty acids and lipid peroxidation. Eur J Clin Nutr 57, 1052-1059.

50. Hagve TA, Lie O \& Grønn M (1993) The effect of dietary $N-3$ fatty acids on osmotic fragility and membrane fluidity of human erythrocytes. Scand J Clin Lab Invest suppl, 215, $75-84$.

51. Harris W, Pottala J, Sands SA, et al. (2007) Comparison of the effects of fish and fish- oil capsules on the $n$ - 3 fatty acid content of blood cells and plasma phospholipids. Am J Clin Nutr 86, 1621-1625.

52. Helland IB, Saugstad OD, Saarem K, et al. (2006) Supplementation of $n$-3 fatty acids during pregnancy and lactation reduces maternal plasma lipid levels and provides DHA to the infants. J Matern Fetal Neonatal Med 19, $397-406$

53. Higgins S, Carroll YL, McCarthy SN, et al. (2001) Susceptibility of LDL to oxidative modification in healthy volunteers supplemented with low doses of $n-3$ polyunsaturated fatty acids. Br J Nutr 85, 23-31.

54. Hodge J, Sanders K \& Sinclair AJ (1993) Differential utilization of eicosapentaenoic acid and docosahexaenoic acid in human plasma. Lipids 28, 525-531.

55. Hoffman DR, Theuer RC, Castañeda YS, et al. (2004) Maturation of visual acuity is accelerated in breast-fed term infants fed baby food containing DHA-enriched egg yolk. $J$ Nutr 134, 2307-2313.

56. Itomura M, Hamazaki K, Sawazaki S, et al. (2005) The effect of fish oil on physical aggression in schoolchildren: a randomized, double-blind, placebo-controlled trial. J Nutr Biochem 16, 163-171.

57. Katan MB, Deslypere JP, van Birgelen AP, et al. (1997) Kinetics of the incorporation of dietary fatty acids into serum cholesteryl esters, erythrocyte membranes, and adipose tissue: an 18-month controlled study. I Lipid Res 38 2012-2022

58. Kew S, Mesa MD, Tricon S, et al. (2004) Effects of oils rich in eicosapentaenoic and docosahexaenoic acids on immune cell composition and function in healthy humans. $A m$ J Clin Nutr 79, 674-681.

59. Khan F, Elherik K, Bolton-Smith C, et al. (2003) The effects of dietary fatty acid supplementation on endothelial function and vascular tone in healthy subjects. Cardiovasc Res 59, 955-962.

60. Laidlaw M \& Holub BJ (2003) Effects of supplementation with fish oil-derived $n-3$ fatty acids and gamma-linolenic acid on circulating plasma lipids and fatty acid profiles in women. Am J Clin Nutr 77, 37-42.

61. Mann NJ, O'Connell SL, Baldwin KM, et al. (2010) Effects of seal oil and tuna-fish oil on platelet parameters and plasma lipid levels in healthy subjects. Lipids 45, 669-681.

62. Mantzioris E, James MJ, Gibson RA, et al. (1994) Dietary substitution with an alpha-linolenic acid-rich vegetable oil increases eicosapentaenoic acid concentrations in tissues. Am J Clin Nutr 59, 1304-1309.

63. McDaniel JC, Ahijevych K \& Belury M (2010) Effect of $n-3$ oral supplements on the $n-6 / n-3$ ratio in young adults. West I Nurs Res 32, 64-80.

64. Miles EA, Banerjee T \& Calder PC (2004) The influence of different combinations of gamma-linolenic, stearidonic and eicosapentaenoic acids on the fatty acid composition of blood lipids and mononuclear cells in human volunteers Prostaglandins Leukot Essent Fatty Acids 70, 529-538.

65. Mills DE, Murthy M \& Galey WR (1995) Dietary fatty acids, membrane transport, and oxidative sensitivity in human erythrocytes. Lipids 30, 657-663.

66. Milte CM, Coates AM, Buckley JD, et al. (2008) Dosedependent effects of docosahexaenoic acid-rich fish oil on erythrocyte docosahexaenoic acid and blood lipid levels Br J Nutr 99, 1083-1088.

67. Minns LM, Kerling EH, Neely MR, et al. (2010) Toddler formula supplemented with docosahexaenoic acid (DHA) improves DHA status and respiratory health in a randomized, double-blind, controlled trial of US children less than 3 years of age. Pro LeukoEs Fat Acids 82, 287-293.

68. Montgomery C, Speake BK, Cameron A, et al. (2003) Maternal docosahexaenoic acid supplementation and fetal accretion. Br J Nutr 90, 135-145.

69. Murphy KJ, Meyer BJ, Mori TA, et al. (2007) Impact of foods enriched with $n$-3 long-chain polyunsaturated fatty acids on erythrocyte $n-3$ levels and cardiovascular risk factors. $\mathrm{Br} \mathrm{J}$ Nutr 97, 749-757.

70. Neubronner J, Schuchardt JP, Kressel G, et al. (2011) Enhanced increase of omega-3 index in response to longterm $n-3$ fatty acid supplementation from triacylglycerides versus ethyl esters. Eur J Clin Nutr 65, 247-254.

71. Otto SJ, van Houwelingen AC \& Hornstra G (2000) The effect of different supplements containing docosahexaenoic acid on plasma and erythrocyte fatty acids of healthy nonpregnant women. Nutr Res 20, 917-927.

72. Otto SJ, van Houwelingen AC \& Hornstra G (2000) The effect of supplementation with docosahexaenoic and arachidonic acid derived from single cell oils on plasma and erythrocyte fatty acids of pregnant women in the second trimester Prostaglandins Leukot Essent Fatty Acids 63, 323-328.

73. Palozza P, Sgarlata E, Luberto C, et al. (1996) n-3 Fatty acids induce oxidative modifications in human erythrocytes depending on dose and duration of dietary supplementation. Am J Clin Nutr 64, 297-304. 
74. Park Y \& Harris W (2002) EPA, but not DHA, decreases mean platelet volume in normal subjects. Lipids 37, 941-946.

75. Rees D, Miles EA, Banerjee T, et al. (2006) Dose-related effects of eicosapentaenoic acid on innate immune function in healthy humans: a comparison of young and older men. Am J Clin Nutr 83, 331-342.

76. Sanders TAB, Gleason K, Griffin B, et al. (2006) Influence of an algal triacylglycerol containing docosahexaenoic acid (22:6n-3) and docosapentaenoic acid (22:5n-6) on cardiovascular risk factors in healthy men and women. $\mathrm{Br} J \mathrm{Nutr}$ 95, 525-531.

77. Sanjurjo P, Ruiz-Sanz JI, Jimeno P, et al. (2004) Supplementation with docosahexaenoic acid in the last trimester of pregnancy: maternal-fetal biochemical findings. $J$ Perinat Med 32, 132-136.

78. Smuts CM, Borod E, Peeples JM, et al. (2003) High-DHA eggs: feasibility as a means to enhance circulating DHA in mother and infant. Lipids 38, 407-414.

79. Sørensen NS, Marckmann P, Høy CE, et al. (1998) Effect of fish-oil-enriched margarine on plasma lipids, lowdensitylipoprotein particle composition, size, and susceptibility to oxidation. Am J Clin Nutr 68, 235-241.

80. Stark KD, Park EJ, Maines VA, et al. (2000) Effect of a fish-oil concentrate on serum lipids in postmenopausal women receiving and not receiving hormone replacement therapy in a placebo-controlled, doubleblind trial. Am J Clin Nutr 72, 389-394.

81. Surai PF, MacPherson A, Speake BK, et al. (2000) Designer egg evaluation in a controlled trial. Eur J Clin Nutr 54, 298-305.

82. Thies F, Nebe-von-Caron G, Powell JR, et al. (2001) Dietary supplementation with gamma-linolenic acid or fish oil decreases $\mathrm{T}$ lymphocyte proliferation in healthy older humans. J Nutr 131, 1918-1927.

83. Vognild E, Elvevoll EO, Brox J, et al. (1998) Effects of dietary marine oils and olive oil on fatty acid composition, platelet membrane fluidity, platelet responses, and serum lipids in healthy humans. Lipids 33, 427-436.

84. Wallace JM, McCabe AJ, Robson PJ, et al. (2000) Bioavailability of $n-3$ polyunsaturated fatty acids (PUFA) in foods enriched with microencapsulated fish oil. Ann Nutr Metab 44, 157-162.

85. Yaqoob P, Pala HS, Cortina-Borja M, et al. (2000) Encapsulated fish oil enriched in alpha-tocopherol alters plasma phospholipid and mononuclear cell fatty acid compositions but not mononuclear cell functions. Eur J Clin Invest 30, $260-274$. 